\title{
El difícil camino desde el diseño a la implementación de las reformas de salud
}

\author{
OSCAR ARTEAGA ${ }^{(1)}$
}

La propuesta del Gobierno para la reforma del sector salud se expresa en un conjunto de proyectos de ley que fueron presentados al parlamento el año 2002, siendo el proyecto que define un Plan de Acceso Universal con Garantías Explícitas (AUGE), el núcleo de la propuesta de cambio. De este conjunto de proyectos, sólo el de autoridad sanitaria y hospitales autogestionados ha terminado su trámite legislativo para llegar a transformarse en la Ley 19.937. A pesar que el grado de avance del resto de los proyectos en la discusión legislativa es variable, la publicación de la Ley de Autoridad Sanitaria y la puesta en marcha de los pilotos AUGE implica que se transita desde la etapa de diseño a la de implementación.

La sistematización de la experiencia de los procesos de reforma en otros países, enseña que los diseñadores de las propuestas de reforma asignan mucha más importancia al diseño y al contenido de las reformas que a los procesos de implementación ${ }^{1,2}$. En no pocas ocasiones se estima que la implementación no es más que una consecuencia natural del diseño, la fase en la cual el equipo de la línea se hace cargo de llevar a la práctica el modelo que le entrega el equipo de diseñadores. Estas experiencias también enseñan que es en la implementación donde se determina el éxito o fracaso de las propuestas de reforma.

Anteriores artículos en ésta y otras publicaciones ${ }^{3,4}$ han expresado opiniones diversas respecto del diseño por el cual optó el equipo del Gobiemo para llevar a cabo la reforma sectorial que explicita garantías de acceso y protección financiera para un conjunto de problemas de salud. El presente artículo tiene como objetivo analizar algunos elementos de la etapa de implementación que se ha inaugurado, de manera de contribuir a un debate que pueda ser útil para quienes ocupan posiciones de responsabilidad decisional.

\section{CONTRIBUCIONES Y DIFICULTADES EN LA IMPLEMFNTACIÓN DEL AUGE}

Desde la Escuela de Salud Pública, junto con valorar el esfuerzo realizado por el equipo técnico del Ministerio de Salud para definir el Plan AUGE, se ha destacado la enorme trascendencia que tiene poner al ciudadano como eje central del cambio que se quiere impulsar. El aumentar el poder ciudadano fortaleciendo el control social sobre las instituciones de salud tiende a atenuar las asimetrías entre el usuario de servicios de salud y los prestadores individuales e institucionales de atención. Esta es, sin duda, la contribución más significativa de la actual reforma de salud. Consistentemente con lo anterior, es también valorable que las garantías de oportunidad de acceso, calidad y protección financiera establecidas en el Plan sean obligatorias para los sistemas de salud público y privado.

La discusión del financiamiento asociado con el Plan AUGE ha permitido visualizar dimensiones que hasta ahora no habían podido plantearse con fuerza, especialmente respecto a la naturaleza de la cotización para salud. Esta discusión está desnudando la diferencia entre la situación chilena actual y la concepción de seguridad social que se tiene en países desarrollados 5 y que forma parte de las raíces de la seguridad social chilena ${ }^{6}$. Este tema es abordado en profundidad en el artículo del Dr. Marcos Vergara en este mismo número de la Revista Chilena de Salud Pública. Igualmente relacionado con el financiamiento del Plan, la propuesta de incrementar los impuestos al alcohol, tabaco

("Escuela de Salud Pública. Universidad de Chilc. oarteaga $@$ med uchile cl 
y diesel fue bienvenida desde una perspectiva de Salud Pública, pues estas sustancias son dañinas para la salud y al aumentar el costo de ellas, se reduce su consumo.

AUGE considera un conjunto de 56 problemas de salud definidos de acuerdo a objetivos sanitarios. Sin embargo, la lógica epidemiológica del diseño por el que se optó no necesariamente considera en forma suficiente las realidades ligadas a la naturaleza de los servicios de salud, lo que transforma la alternativa adoptada por el Gobierno en una opción que es compleja de implementar y administrar.

Más allá de la complejidad del diseño, una restricción muy importante para operacionalizar el Plan AUGE es la limitada disponibilidad de información, tema que reviste la mayor importancia, pues tiene impactos en el dimensionamiento de costos y de servicios de salud. Una mirada positiva sobre este tema es que la implementación de AUGE debiera ir generando una tensión que estimule el mejoramiento de los sistema de información, pues el uso de la información es un poderoso incentivo para mejorar la calidad, disponibilidad y oportunidad de ésta. El desafío, sin embargo, es considerable, pues los requerimientos de información son muy importantes a nivel del paciente individual y a nivel agregado, para el manejo clínico y financiero, así como para el monitoreo, control y evaluación. El horizonte de tiempo en el que se requiere esta información hace difícil que los actuales sistemas logren adaptarse con la velocidad necesaria. Una expresión de la debilidad de los sistemas de información es que el inicio de los pilotos AUGE ha significado niveles de recursos mucho mayores a las estimaciones previas.

Debido a la restricción determinada por necesidades de las personas que son mayores a la disponibilidad de recursos, es fácil entender que resulta necesario priorizar y racionalizar, lo cual no significa que ésta sea una tarea fácil y exenta de controversia ${ }^{7}$. La discusión conceptual respecto a llevar la priorización de problemas de salud hasta el nivel del individuo excede el objetivo de este artículo. Sin embargo, cabe señalar que la manera inicial de comunicar el Plan significó que se interpretara como una propuesta de canasta que excluiría las prestaciones no consideradas en AUGE, aspecto que ha dado base para la campaña de resistencia que han desarrollado los gremios del sector.
La implementación del Plan garantizado tendrá que ir lidiando con el hecho que la atención de salud supone la movilización de recursos que, con pocas excepciones, son polivalentes y participan de la respuesta a múltiples problemas de salud. Por lo mismo, existe el riesgo de limitar el uso de estos recursos a un cierto número de enfermedades prioritarias, o dicho de otro modo, que en la práctica se excluya de su beneficio a una cierta cantidad de población que no padece las enfermedades priorizadas. Se puede entrar aquí en una contradicción social, pues la enfermedad de las personas podría transformarse en el criterio de exclusión. Para el ciudadano común puede resultar muy difícil entender por qué algunas enfermedades dan derecho a una atención y otras no. La propia experiencia de AUGE enseña que resulta casi imposible para la autoridad, resistir la presión de grupos de enfermos para incorporar un determinado problema de salud en el plan garantizado, por rara o poco frecuente que sea su patología.

Como ha señalado la autoridad política del sector en reiteradas oportunidades, la implementación de AUGE requiere otros cambios considerados en los distintos proyectos del paquete de reformas de salud. En este sentido, la reciente aprobación de la Ley 19.937 , de autoridad sanitaria y hospitales autogestionados, hace varias contribuciones. Es indudable que contribuye a ordenar el sector salud, fortaleciendo la autoridad sanitaria regional al transferir a las Secretarías Regionales Ministeriales varias de las funciones regulatorias que actualmente desarrollan las Direcciones de Servicios de Salud. Esta ley también significa un gran avance en el tema de entregar mayores espacios de libertad para la gestión de los hospitales públicos.

Siendo correcto el movimiento de fortalecer la capacidad regulatoria de la autoridad sanitaria que se establece en la Ley 19.337 (para las Secretarías Regionales Ministeriales y Superintendencia de Salud), conviene tener presente las dificultades prácticas asociadas al ejercicio de la regulación, con el fin de modular expectativas. En efecto, existen al menos dos órdenes de dificultades para ejercer la regulación: asimetría de información entre regulador y regulado ${ }^{8}$; y costos administrativos o de transacción. Por una parte, el ejercicio de la regulación requiere información y, en la práctica, los regulados poseen un mejor conocimiento de sus procesos productivos y/o de las condiciones de deman- 
da que ellos enfrentan ${ }^{8}$. De manera más crucial, el grado en que el ejercicio de la regulación es capaz de alcanzar objetivos sociales depende de la información que posea el regulador. Por otra parte, pueden haber importantes costos asociados a la implementación de los mecanismos regulatorios". En la práctica, el proceso de implementación de la transferencia de las funciones de autoridad sanitaria desde las Direcciones de Servicios de Salud a las SEREMI, pareciera estar evidenciando que la transferencia de funciones no necesariamente significa que se transfieran los recursos para ejercerlas. Existe el riesgo, entonces, que las nuevas funciones no puedan ser ejercidas por la nueva instancia y dejen de ser ejercida por la anterior, situación que se ha reportando en otros procesos de reforma ${ }^{10}$.

Un aspecto débil de la nueva ley es que no logra resolver con claridad el tema de la instancia coordinadora de la red asistencial, pues se fortalece el rol del Fondo Nacional de Salud (FONASA) actuando directamente con los hospitales autogestionados. En la práctica, este fortalecimiento se hace a expensas del debilitamiento de las atribuciones del coordinador de la red (función asignada a la Dirección de Servicio de Salud). Esto permite vislumbrar que se afectarán las posibilidades reales de hacer una adecuada gestión de la demanda con criterio sanitario. La determinación de necesidades de atención de salud no es una función que competa a FONASA, ni tampoco corresponde exigirla a las direcciones de los hospitales. A estas últimas se les debe exigir eficiencia en el uso de los recursos que se les entregan, pero es otra la instancia que debe definir el tipo, cantidad y calidad de las prestaciones que se deben entregar a la población. La articulación de la respuesta de la oferta a las necesidades de atención, que previamente ha debido definir, es la tarea de la instancia coordinadora de la red.

El ejercicio de capacidad gestora de red por parte de las actuales Direcciones de Servicios de Salud es actualmente limitado. El diseño planteado en la Ley 19.937 y el desarrollo de las etapas iniciales de implementación no facilita el que estas capacidades se puedan desarrollar y fortalecer. En este mismo sentido, al crearse una nueva instancia central, la Subsecretaría de Redes Asistenciales, tiene el riesgo de favorecer una mayor intervención del nivel central en la gestión local, disminu- yendo aún más las atribuciones que en la práctica pueda ejercer la coordinación de la red asistencial.

Mientras se discutían los proyectos en el Congreso, el ejecutivo puso en marcha los pilotos del Plan AUGE, lo que demuestra su voluntad política por sacar adelante la reforma. Es probable que aún sea muy precoz para realizar evaluaciones de estos esfuerzos. Algunas evaluaciones no sistemáticas destacan los positivos efectos asociados al manejo de algunos de los problemas considerados en AUGE (cáncer infantil y terapia anti SIDA, por ejemplo), mientras otras destacan incrementos en las listas de espera de pacientes con algunos otros problemas considerados en AUGE (insuficiencia renal crónica, por ejemplo) ${ }^{11}$ o con problemas no considerados en AUGE. Con todo, la implementación gradual de AUGE permite ir visualizando los problemas que surgen al avanzar del diseño a la implementación e ir haciendo las adecuaciones que se requieran. En este sentido, la extensión del plazo hasta que AUGE se encuentre en régimen es una decisión responsable y prudente por parte de las máximas autoridades del sector. La manera en que algunos prestadores hospitalarios están respondiendo a las señales que se han estado dando al ir implementando los planes pilotos, obligan a estar atento para adoptar estrategias que prevengan estos riesgos, especialmente el traslado de listas de espera desde problemas AUGE a problemas no AUGE.

No puede dejar de destacarse que la autoridad haya recurrido a las universidades para procurar acopiar la mejor evidencia sobre la efectividad y costo de las intervenciones consideradas en el abordaje de los problemas priorizados en AUGE. Este esfuerzo está mostrando que la evidencia actualmente disponible en las bases de datos de los centros internacionales dedicados seriamente a este tema, es bastante menor que lo que normalmente se piensa. Por lo mismo, el nivel de incertidumbre a la hora de tomar decisiones en el contexto del diseño de plan garantizado por el que se optó seguirá siendo alto. Un aspecto relacionado con este mismo punto es el establecimiento de algoritmos y protocolos de atención, materia en la cual la autoridad sectorial también ha estado haciendo esfuerzos. La evidencia muestra que muchos problemas de salud cuentan con protocolos o guías de manejo clínico, pero que en la práctica éstos no se siguen. En este sentido, un elemento tanto o más 
importante que los contenidos específicos de las guías, es que los profesionales que deben llevarlos a la práctica se sientan comprometidos con ellos, lo que se facilita si han sido parte del proceso de construcción de los mismos. Aunque también éste es un tema cuya evaluación puede tener lecturas distintas, en los discursos de las sociedades científicas parecería predominar un reclamo por su escasa participación.

\section{A MODO DE CONCLUSIONES PRELIMINARES}

Es de justicia valorar en todo su mérito el hecho que, desde que el país recuperó su democracia en 1990, no se había contado con la voluntad política del Gobiemo para priorizar el sector salud, y realizar al interior de éste, esfuerzos de transformación tan significativos como los planteados en los proyectos del paquete de reformas del Presidente Lagos.

Los temas que abordan los diferentes proyectos se encuentran en sintonía con importantes problemas del sector salud, y que constituyen parte de un diagnóstico compartido por muchos actores. Las soluciones planteadas, sin embargo, resultan controversiales. Esto último es esperable, toda vez que en un sistema complejo como el sistema de salud coexisten múltiples actores con intereses diversos y, muchas veces, contrapuestos. En este sentido, el esfuerzo gubernamental por situar a los usuarios de los servicios de salud como eje de la propuesta de reforma, mediante un discurso e instrumentos que intentan aumentar el poder cilldadano para favorecer un mayor control social, es de mucha trascendencia.

La perseverancia de este impulso en el tiempo debiera redundar en cambios en la cultura que predomina al interior del sector salud. Las sociedades organizan sistemas de salud para responder a las necesidades de las personas y comunidades, y son estas necesidades las que deben alinear los intereses de otros actores, sean estos prestadores o aseguradores. El espíritu de la decisión del Gobierno por explicitar derechos y un plan garantizado que pueda asegurarse a todas las personas sin discriminaciones se orienta en la dirección correcta. La opción de diseño por la que se optó, sin embargo, es compleja y difícil de operacionalizar. La información con que se cuenta para implementar AUGE es precaria en cantidad, calidad y oportunidad, lo que ya se empieza a evidenciar en las fases iniciales de implementación. Sólo en el mediano plazo, cuando AUGE se encuentre operando en régimen y las personas enfrenten la experiencia de ser incluidos o excluidos en los beneficios del plan, se podrá conocer el grado de tolerancia de la sociedad chilena a un diseño de mínimos garantizables. Si las raíces de la seguridad social europea con que organizó el sector salud chileno siguen presentes en nuestra sociedad, no es descartable que la tolerancia a las exclusiones no sea alta.

Como se ha señalado, la sistematización de la experiencia de los procesos de reforma en otros países, enseña que el tránsito desde el nivel de diseño de ésta hasta la implementación de los cambios es generalmente más largo y difícil de lo que imaginan los diseñadores. Por los antecedentes que empiezan a acumularse en el caso chileno, pareciera que se reproduce esta dinámica. La implementación de los cambios derivados del diseño trasunta un proceso extremadamente desgastador. Por lo mismo, es la hora de aunar esfuerzos para que, más allá de las legítimas diferencias que se hayan tenido en la fase de diseño de la reforma, los diversos actores técnicos, políticos y gremiales y sociales puedan sentarse a dialogar y buscar caminos de entendimiento y solución a los problemas sobre la base de objetivos de bienestar social para todos los chilenos.

\section{REFERENCIAS}

1.- WALT G GILSON L. Reforming the health sector in developing countries: the central role of policy analysis. Health Policy and Planning 1994; 9 (4): 353-70.

2.- WHO. European Health Care Reform: Analysis of current strategies. World Health Organization Regional Office for Europe. WHO regional publications. Europe series; No. 72. Copenhagen: WHO 1997.

3.- ESCUELA DE SALUD PÚBLICA. La Reforma de la Salud en Chile desde una perspectiva de salud pública. Revista Chilena de Salud Pública 2002;6 (2-3): $112-8$.

4.- ARTEAGA O. El Plan AUGE y la Reforma de Salud: Todo un cambio cultural. En Cárdenas, J.P., Meza, R. (eds). Anuario de Chile/ Universidad de Chile 2003/4. Santiagode Chile: Universidad de Chile 2003.

5.- BARR N. The Economics of the Welfare State. Third Edition. Oxford: Oxford University Press 
1998.

6.- JIMÉNEZ J, BOSSERT T J. Chile's Health Sector Reform: Lessons from Four Reform Periods. In: Berman, P. (ed.) Health Sector Reform in Developing Countries: Making Health Development Sustainable. Boston: Harvard University Press 1995.

7.- BUTLER J. The ethics of health care rationing: principles and practice. London: Cassell 1999.

8.- PROPPER C. Quasi-markets and regulation. In: Le Grand, J., Bantlett, W. (eds.). Quasi-Markets and Social Policy. Hong Kong: MacMillan Press Lid
1993.

9. KUMARANAYAKE L. The role of regulation: Influencing private sector activity within health sector reform. Journal of International Development . 1997); 9: 641-9.

10.- MILLS A, BENNETT S, RUSSELL S. The Challenge of Health Sector Reform: What Must Governments Do? Hampshire, New York: Anthony Rowe Ltd 2001.

11.- GONZÁLEZ F F. Implementación del plan AUGE en pacientes con IRC. Rev Méd Chile 2003; 131: $545-51$ 